\title{
Reviewer Acknowledgments 2018
}

Published online: 8 February 2019

(C) Springer Nature Switzerland AG 2019

The Editor-in-Chief of the International Journal of Latin American Religions would like to publicly thank the reviewers who have contributed to the journal in Volume 2 (2018) and who have authorized the public disclosure of their names. The expertise and contribution of the reviewers are critical to continually maintain the good standard and the success of our publication.

Agustina Gracia

Alejandro Frigerio

Amurabi Oliveira

Antonio Genivaldo Cordeiro de Oliveira

Brenda Carranza

Catherine Caufield

Cecília Mariz

César Ceriani Cernadas

Cristina Gutièrrez Zuñiga

Daniel M. Murray

Daniela Cordovil Corrêa dos Santos

Dilaine Soares Sampaio

Esther Langdon

Everton de Oliveira Maraldi

Ezer Roboam May May

Fábio Stern

Felipe Luarte Correa

Francisco Diez de Velasco

Frederico Pieper Pires

Gabriel Martino

Hugo Córdova Quero

Jean Paul Sarrazin

José Suárez

Joseph Hill

Leandro Durazzo

Maria Eugenia Funes

Massimo Introvigne

\author{
Mercedes Saizar \\ Michael Winkelman \\ Miriam Raquel Wachholz Strelhow \\ Nahayeilli Juárez-Huet \\ Néstor da Costa \\ Pablo Wright \\ Pamela Siegel \\ Patrícia Rodrigues de Souza \\ Rafael Shoji \\ Regina Yoshie Matsue \\ Robin Wright \\ Rodrigo Wolff Apolloni \\ Ruth Jatziri García Linares \\ Sandra Duarte de Souza \\ Shigeko Mato \\ Silas Guerriero \\ Susana Monreal \\ Suzana Ramos Coutinho \\ Suzanne Newcombe \\ Veronica Gimenez Beliveau \\ Victor Espinosa \\ Vincenzo Pace
}

Publisher's Note Springer Nature remains neutral with regard to jurisdictional claims in published maps and institutional affiliations. 